\title{
Briófitas epífitas de fragmentos de Floresta Atlântica da Reserva Ecológica Michelin, Estado da Bahia, Brasil ${ }^{1}$
}

\author{
Hermeson Cassiano de Oliveira ${ }^{2,4}$ e Cid José Passos Bastos ${ }^{3}$
}

Recebido: 2.07.2013; aceito: 27.05.2014

\begin{abstract}
Epiphytic Bryophytes of Atlantic Forest Fragments of Reserva Ecológica Michelin, Bahia State, Brazil). The Reserva Ecológica Michelin is located in the southern region of Bahia State, Brazil. It has a total area of 3,096 ha and contains a variety of environments from Atlantic Forest such as restinga, mangrove and estuary, and important remnants of rainforest. The floristic survey of epiphytic bryophytes occurring in forest fragments of the Reserva Ecológica Michelin resulted in 206 taxa (195 species and 11 varieties), distributed in 73 genera and 25 families of epiphytic bryophytes. Of the total, 123 are liverworts, distributed in 33 genera and eight families, and 83 mosses belonging to 40 genera and 17 families. We report six new occurrences, being four for the Northeast region of Brazil and two for Bahia State. A complete listing is provided containing the occurrence of species in each fragment, the geographical distribution, worldwide and in Brazil.
\end{abstract}

Keywords: floristic, liverworts, mosses

RESUMO - (Briófitas Epífitas de Fragmentos de Floresta Atlântica da Reserva Ecológica Michelin, Estado da Bahia, Brasil). A Reserva Ecológica da Michelin localiza-se na região sul do Estado da Bahia. Possui uma área total de 3.096 ha e contém uma diversidade de ambientes do complexo Floresta Atlântica, como restinga, manguezal e estuário, além de importantes fragmentos remanescentes de Floresta Ombrófila. O levantamento florístico das espécies de briófitas epífitas ocorrentes nos fragmentos florestais da Reserva Ecológica Michelin resultou em 206 táxons infragenéricos (195 espécies e 11 variedades), distribuídos em 73 gêneros e 25 famílias de briófitas epífitas. Do total, 123 são hepáticas distribuídas em 33 gêneros e oito famílias; e 83 musgos pertencentes a 40 gêneros e 17 famílias. São relatadas sete novas ocorrências, sendo quatro para a região Nordeste do Brasil e duas para o Estado da Bahia. É fornecida uma listagem completa contendo a ocorrência das espécies em cada fragmento, distribuição geográfica mundial e no Brasil.

Palavras-chave: florística, hepáticas, musgos

\section{Introdução}

A flora briofítica é abundante no mundo inteiro, reunindo aproximadamente 15.000 espécies e mais de 1.200 gêneros. Para o Neotrópico, são registradas cerca de 3.980 espécies de briófitas, sendo 2.600 musgos, 1.350 hepáticas e 30 antóceros (Gradstein et al. 2001).

O conhecimento sobre as briófitas brasileiras encontra-se disperso em publicações como catálogos, listas de espécies, algumas flórulas e poucas revisões taxonômicas. O Brasil possui uma brioflora rica, para a qual eram consideradas, até pouco tempo, aproximadamente 1.650 espécies (Yano 1981, 1989, 1995, 2006), correspondendo a cerca de $40 \%$ das espécies citadas para a América tropical (Gradstein et al. 2001, Gradstein \& Costa 2003). No entanto, este número vem decrescendo, em função de diversos trabalhos onde muitas espécies foram sinonimizadas. No mais recente catálogo sobre antóceros e hepáticas do Brasil, Yano (2008) relacionou 1.046 táxons. Com relação aos musgos, Costa et al. (2011) citaram 892 espécies. De acordo com a Lista de Espécies da Flora do Brasil (Forza et al. 2010), aproximadamente 1.521 espécies de briófitas são relatadas atualmente para o país.

1. Parte da Tese de Doutorado do primeiro Autor

2. Universidade Estadual do Piauí, Campus Heróis do Jenipapo, Laboratório de Biologia Vegetal, Av. Sto. Antônio s/n, 64280-000 Campo Maior, PI, Brasil

3. Universidade Federal da Bahia, Instituto de Bilologia, Departamento de Botânica, Laboratório de Taxonomia de Briófitas, Campus Ondina, 40170-280 Salvador, BA, Brasil

4. Autor para correspondência: hermeson123@gmail.com 
As briófitas do Domínio Floresta Atlântica têm sido amplamente estudadas nos últimos anos, principalmente nas regiões Sul e Sudeste do Brasil, as quais possuem o maior número de estudos: Angely (1961, 1968), Sehnem (1969, 1970, 1972, 1976, 1978, 1979, 1980), Kummorow \& Prevedello (1982), Giacontti \& Vittal (1989), Schäfer-Verwimp (1989, 1991, 1992), Costa \& Yano (1995), Hirai et al. (1998), Oliveira-e-Silva \& Yano (2000a, b), Visnadi \& Vital (2000), Yano \& Colletes (2000), Visnadi (2002, 2005, 2006), Costa \& Silva (2003), Costa et al. (2005), Yano (2005). Na região Nordeste, a maioria dos trabalhos está concentrada nos Estados da Bahia e Pernambuco: Pôrto (1990), Marinho \& Mariz (1992), Germano \& Pôrto (1996), Vilas Bôas-Bastos \& Bastos (2000, 2002), Bastos \& Yano (2002, 2004), Bastos et al. (2003), Valente \& Pôrto (2006a, b), Vilas Bôas-Bastos (2009), Bastos (2011). Apesar do acentuado estágio de degradação da Floresta Atlântica, as publicações referentes à brioflora neste ecossistema confirmam uma riqueza brioflorística elevada (Oliveira-e-Silva et al. 2002).

Os estudos referentes às briófitas de Floresta Atlântica do Estado da Bahia cresceram consideravelmente em quantidade nos últimos anos, apresentando relevantes resultados com relação à riqueza e à diversidade de espécies no Estado, incluindo novos táxons para a ciência (Vilas Bôas-Bastos \& Bastos 2000, 2002, Bastos \& Yano 2002, 2004, 2005, 2009, Bastos \& Gradstein 2006, Valente \& Pôrto 2006a, b, Vilas Bôas-Bastos 2009).

O presente trabalho teve como objetivo o levantamento florístico das espécies de briófitas epífitas ocorrentes nos fragmentos florestais da Reserva Ecológica Michelin (REM), no Estado da Bahia, além de verificar a distribuição geográfica das espécies no Brasil e no mundo.

\section{Material e métodos}

As coletas foram realizadas na Reserva Ecológica da Michelin (figura 1) na região sul do Estado da Bahia (134ㅇ' $\left.08^{\prime \prime} \mathrm{S}, 39^{\circ} 10^{\prime} 03^{\prime \prime} \mathrm{W}\right)$, no período de 2009 a 2011 , com duração mínima de três dias cada, perfazendo um total de oito excursões de campo. A área total da reserva é de 3.096 ha e contém uma diversidade de ambientes do complexo Floresta Atlântica como restinga, manguezal e estuário, além de importantes fragmentos remanescentes de floresta ombrófila (K. Flescher, dados não publicados).
A região tem clima Tropical úmido (Af) com precipitação anual de $2.051 \mathrm{~mm}$, com chuvas distribuídas ao longo do ano e temperaturas médias variando entre 18 e $30^{\circ} \mathrm{C}$ (CEI/CONDER 1993); apresenta exuberância paisagística associada aos ambientes florestal e aquático, tanto marinho como continental dulciaquícola, estando relacionada a este último a maior queda d'água do litoral brasileiro, a Cachoeira da Pancada Grande com $62 \mathrm{~m}$ de altura que se constitui em significativo atrativo turístico, inserido na área da Reserva.

A Floresta ombrófila pertencente à Reserva está localizada em áreas do município de Igrapiúna formando mosaicos com os seringais, distribuída em quatro fragmentos: Mata de Pacangê (PAC) - 550 ha, Mata da Vila Cinco (MV) - 180 ha, Mata de Pancada Grande (PG) - 172 ha e Mata do Luis Inácio (LI) - 140 ha, com uma cobertura total de 1.042 ha dentro do Corredor Central da Floresta Atlântica; sua altitude varia entre 160 e 327 m; é classificada, segundo Veloso et al. (1991), como Floresta Ombrófila Densa de Terras Baixas.

As amostras coletadas foram identificadas usando-se chaves de identificação e ilustrações presentes em bibliografia especializada como Fulford (1963, 1966, 1968, 1976), Florschütz (1964), Sharp et al. (1994), Buck (1998), Gradstein et al. (2001), Visnadi (2002), Dauphin (2003) e Gradstein \& Costa (2003). Além disso, foram feitas comparações com materiais de herbário previamente identificados e consultas aos especialistas de determinados grupos. Amostras depositadas no Herbário Alexandre Leal Costa (ALCB) da Universidade Federal da Bahia

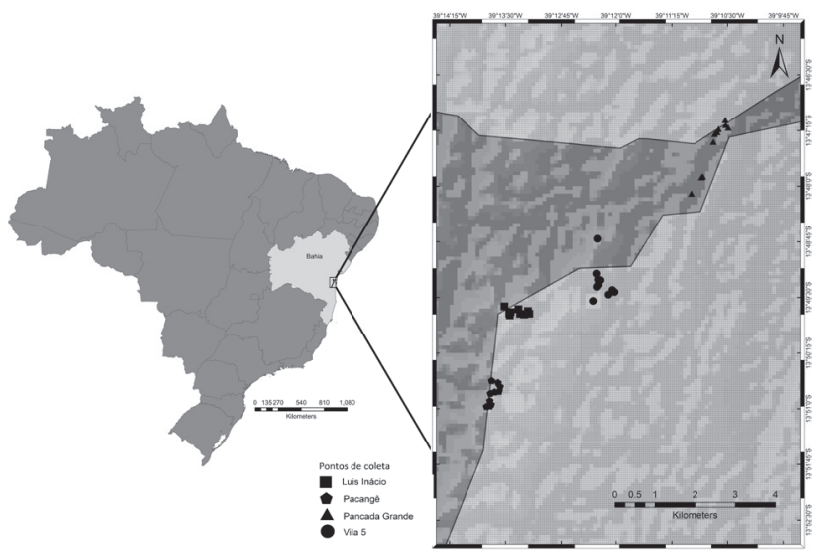

Figura 1. Localização da Reserva Ecológica Michelin no Estado da Bahia, Brasil e dos pontos de coleta nos quatro fragmentos de Floresta Atlântica.

Figure 1. Reserva Ecológica Michelin location in Bahia State, Brazil and the collection points in the four forest fragments of Atlantic Forest. 
Tabela 1. Briófitas epífitas da Reserva Ecológica Michelin, Igrapiúna, Estado da Bahia, Brasil. Fragmentos. MV: Vila 5; PG: Pancada grande; LI: Luís Inácio; PAC: Pacangê.

Table 1. Epiphytic bryophytes of Reserva Ecológica Michelin, Igrapiúna, Bahia State, Brazil. Fragments. MV: Vila 5; PG: Pancada Grand; LI: Luís Inácio; PAC: Pacangê.

\begin{tabular}{|c|c|c|c|c|c|c|}
\hline Táxons & MV & PG & LI & $\mathrm{PAC}$ & $\begin{array}{c}\text { Distribuição } \\
\text { mundial }\end{array}$ & Distribuição no Brasil \\
\hline \multicolumn{7}{|l|}{$\begin{array}{l}\text { MARCHANTIOPHYTA } \\
\text { ANEURACEAE }\end{array}$} \\
\hline Riccardia chamedryfolia (With.) Grolle & & & + & + & Neotropical & BA, ES, RJ, SP. \\
\hline \multicolumn{7}{|l|}{ FRULLANIACEAE } \\
\hline Frullania apiculata (Reinw., Blume \& Nees) Nees & & & & + & Neotropical & AM, BA, PA, PE, RJ, SP. \\
\hline Frullania atrata $(\mathrm{Sw}$.$) Nees$ & + & & & & Neotropical & $\begin{array}{l}\text { BA, ES, PA, PE, RJ, RS, } \\
\text { SP. }\end{array}$ \\
\hline Frullania caulisequa (Nees) Nees & + & + & + & + & Neotropical & $\begin{array}{l}\text { AC, BA, ES, MG, PA, PE, } \\
\text { RJ, RS, SC, SE, SP. }\end{array}$ \\
\hline Frullania kunzei Lehm. \& Lindenb. & + & & + & & Neotropical & AL, GO, PA, RJ, SC, SP. \\
\hline \multicolumn{7}{|l|}{ LEJEUNEACEAE } \\
\hline $\begin{array}{l}\text { Acanthocoleus aberrans (Lindenb. \& Gottsche) } \\
\text { Kruijt }\end{array}$ & & & + & & Pantoprical & $\begin{array}{c}\mathrm{BA}, \mathrm{GO}, \mathrm{MG}, \mathrm{PR}, \mathrm{RJ}, \mathrm{RS}, \\
\text { SC. }\end{array}$ \\
\hline $\begin{array}{l}\text { Aphanolejeunea microscopica var. exigua } \\
\text { (A. Evans) Bernecker \& Pócs }\end{array}$ & & & & + & Neotropical & BA, SP. \\
\hline Archilejeunea auberiana (Mont.) A. Evans & + & + & + & & Neotropical & $\begin{array}{c}\mathrm{AC}, \mathrm{AM}, \mathrm{BA}, \mathrm{PA}, \mathrm{RR}, \\
\text { RS, SP. }\end{array}$ \\
\hline $\begin{array}{l}\text { Archilejeunea fuscescens (Hampe ex Lehm.) } \\
\text { Fulford }\end{array}$ & + & + & + & + & Neotropical & $\mathrm{AC}, \mathrm{AM}, \mathrm{BA}, \mathrm{PA}, \mathrm{PE}, \mathrm{RR}$. \\
\hline Archilejeunea parviflora (Nees) Steph. & & & + & & Neotropical & $\begin{array}{l}\text { AC, A M, BA, ES, MG, } \\
\text { PA, PE, RJ, RO, RR, SP. }\end{array}$ \\
\hline Bryopteris diffusa (Sw.) Nees & + & & & + & Neotropical & $\begin{array}{l}\text { AL, AM, BA, MG, MT, } \\
\text { PA, PE, RJ, RS, SC, SP. }\end{array}$ \\
\hline Bryopteris filicina (Sw.) Nees & & & & + & Neotropical & $\begin{array}{c}\text { AM, BA, CE, ES, GO, } \\
\text { MG, MT, PA, PR, RJ, RR, } \\
\text { RS, SC, SP. }\end{array}$ \\
\hline Ceratolejeunea cerantha (Nees \& Mont.) Steph. & & & & + & Neotropical & A M, BA, MG, RJ, SP. \\
\hline Ceratolejeunea coarina (Gottsche) Schiffn. & & + & & + & Neotropical & $\begin{array}{l}\text { AC, AM, AP, BA, MA, } \\
\text { PA, PR, SP. }\end{array}$ \\
\hline Ceratolejeunea confusa R.M. Schust. & & + & + & + & Neotropical & AM, BA, PA, PE, SP. \\
\hline Ceratolejeunea cornuta (Lindenb.) Steph. & + & + & + & + & Neotropical & $\begin{array}{l}\text { AM, AP, BA, PA, PE, PR, } \\
\text { RR, RJ, RO, SC, SP. }\end{array}$ \\
\hline Ceratolejeunea cubensis (Mont.) Schiffn. & & + & + & + & Neotropical & $\begin{array}{l}\text { AC, AM, BA, PE, PR, RJ, } \\
\text { RO, SP. }\end{array}$ \\
\hline Ceratolejeunea fallax (Lehm. \& Lindenb.) Bonner & + & & + & + & Neotropical & $\begin{array}{l}\text { AM, BA, MG, PE, RJ, } \\
\text { RO, SP. }\end{array}$ \\
\hline Ceratolejeunea laetefusca (Austin) R.M. Schust. & + & + & + & & Neotropical & $\begin{array}{l}\text { AC, AM, BA, ES, MG, } \\
\text { PA, PE, RJ, SP. }\end{array}$ \\
\hline Ceratolejeunea minuta G. Dauphin & + & + & + & + & Neotropical & BA, SP. \\
\hline
\end{tabular}


Tabela 1 (continuação)

\begin{tabular}{|c|c|c|c|c|c|c|}
\hline Táxons & MV & PG & LI & PAC & $\begin{array}{l}\text { Distribuição } \\
\text { mundial }\end{array}$ & Distribuição no Brasil \\
\hline Ceratolejeunea rubiginosa Gotsche ex Steph. & & + & & + & Neotropical & $\overline{\mathrm{AP}, \mathrm{BA}, \mathrm{CE}, \mathrm{PA}, \mathrm{RJ}, \mathrm{SP} .}$ \\
\hline Cheilolejeunea acutangula (Nees) Grolle & + & + & + & + & Neotropical & $\begin{array}{l}\text { AM, BA, ES, MG, MT, } \\
\text { PE, RJ, SP. }\end{array}$ \\
\hline $\begin{array}{l}\text { Cheilolejeunea adnata (Kunze ex Lehm.) Grolle } \\
\text { var. adnata }\end{array}$ & & & & + & Neotropical & $\begin{array}{l}\text { AM, BA, MT, PA, PE, PR, } \\
\text { SC, SP. }\end{array}$ \\
\hline Cheilolejeunea clausa (Nees \& Mont.) R.M Schust. & + & + & + & & Neotropical & $\begin{array}{l}\text { AM, BA, MG, PA, PR, } \\
\text { RJ, SP. }\end{array}$ \\
\hline $\begin{array}{l}\text { Cheilolejeunea discoidea (Lehm. \& Lindenb.) Kachr. } \\
\text { \& R.M. Schust. }\end{array}$ & + & + & + & + & Neotropical & BA, ES, MG, MT, SP. \\
\hline Cheilolejeunea exinnovata E.W. Jones & + & & & & Neotropical & $\begin{array}{l}\text { AL, AM, BA, CE, ES, PA, } \\
\text { RR, SP. }\end{array}$ \\
\hline $\begin{array}{l}\text { Cheilolejeunea holostipa (Spruce) Grolle \& } \\
\text { R.L. Zhu }\end{array}$ & + & & & & Neotropical & $\mathrm{AL}, \mathrm{BA}, \mathrm{PA}, \mathrm{RJ}, \mathrm{SP}$. \\
\hline Cheilolejeunea inflexa (Hampe ex Lehm.) Grolle & & & & + & Neotropical & BA, SP. \\
\hline Cheilolejeunea intertexta (Lindenb.) Steph. & & & & + & Neotropical & BA. \\
\hline $\begin{array}{l}\text { Cheilolejeunea oncophylla (Ångstr.) Grolle } \\
\text { E. \& Reiner }\end{array}$ & & + & & & Neotropical & BA, MG, PR, RJ, RS, SP. \\
\hline Cheilolejeunea rigidula (Mont.) R.M. Schust. & & & & + & Pantropical & $\begin{array}{c}\mathrm{AC}, \mathrm{AL}, \mathrm{AP}, \mathrm{BA}, \mathrm{ES}, \mathrm{GO}, \\
\mathrm{MA}, \mathrm{MG}, \mathrm{MT}, \mathrm{PA}, \mathrm{PE} \\
\text { PR, RJ, SC, SE, SP. }\end{array}$ \\
\hline Cheilolejeunea sp. & + & + & + & + & & \\
\hline $\begin{array}{l}\text { Cheilolejeunea trifaria (Reinw., Blume \& } \\
\text { Nees) Mizut. }\end{array}$ & + & & & & Pantropical & $\begin{array}{l}\text { AC, AM, BA, ES, MG, } \\
\text { PA, PE, RJ, RR, SP. }\end{array}$ \\
\hline Cheilolejeunea unciloba (Lindenb.) Malombe & + & + & + & + & Pantropical & $\begin{array}{l}\text { BA, ES, PE, RJ, RS, } \\
\text { SC, SP. }\end{array}$ \\
\hline $\begin{array}{l}\text { Cheilolejeunea xanthocarpa (Lehm. \& Lindenb.) } \\
\text { Malombe }\end{array}$ & & + & & & Pantropical & $\begin{array}{l}\text { BA, ES, MG, PE, RJ, } \\
\text { SC, SP. }\end{array}$ \\
\hline Cyclolejeunea chitonia (Taylor ex Lehm.) A. Evans & + & & & + & Neotropical & AP, BA, PA. \\
\hline $\begin{array}{l}\text { Cyclolejeunea convexistipa (Lehm. \& Lindenb.) } \\
\text { A. Evans }\end{array}$ & + & & + & + & Neotropical & AM, AP, BA, PA, SP. \\
\hline Cyclolejeunea luteola (Spruce) Grolle & & & & + & Neotropical & AM, BA, PA, RJ, SP. \\
\hline Diplasiolejeunea brunnea Steph. & & + & + & & Neotropical & $\begin{array}{l}\text { AC, AL, BA, ES, MT, } \\
\text { PA, RJ, RO, SC, SP. }\end{array}$ \\
\hline Drepanolejeunea anoplantha (Spruce) Steph. & & & & + & Neotropical & AM, BA, ES, SP. \\
\hline Drepanolejeunea aracauriae Steph. & & + & & & Neotropical & BA, MG, RS, SC, SP. \\
\hline Drepanolejeunea fragilis Bischl. & & & & + & Neotropical & AM, BA, ES, PE, SP. \\
\hline Drepanolejeunea lichenicola (Spruce) Steph. & & + & & + & Neotropical & BA, PR, SP. \\
\hline Harpalejeunea oxyphylla (Nees \& Mont.) Steph. & + & + & & + & Neotropical & $\begin{array}{l}\text { AM, AP, BA, PA, PE, } \\
\text { PR, RJ, SP. }\end{array}$ \\
\hline Harpalejeunea stricta (Lindenb. \& Gottsche) Steph. & & & + & & Neotropical & BA, PA. \\
\hline Harpalejeunea tridens (Besch. \& Spruce) Steph. & + & & + & + & Neotropical & BA, SP. \\
\hline Lejeunea adpressa Nees & + & & & + & Neotropical & $\mathrm{AC}, \mathrm{BA}, \mathrm{PA}, \mathrm{RJ}, \mathrm{SP}$. \\
\hline
\end{tabular}


Tabela 1 (continuação)

\begin{tabular}{|c|c|c|c|c|c|c|}
\hline Táxons & MV & PG & LI & PAC & $\begin{array}{l}\text { Distribuição } \\
\text { mundial }\end{array}$ & Distribuição no Brasil \\
\hline Lejeunea boryana Mont. & + & + & + & + & Neotropical & AM, BA, PA. \\
\hline Lejeunea caulicalyx (Steph.) E. Reiner \& Goda & + & & & + & Neotropical & $\begin{array}{l}\text { AC, BA, ES, MT, PA, PE, } \\
\text { RJ, SP. }\end{array}$ \\
\hline Lejeunea controversa Gottsche & & & + & + & Neotropical & $\mathrm{AC}, \mathrm{BA}, \mathrm{SP}$ \\
\hline Lejeunea filipes Spruce & + & & + & + & Neotropical & BA. \\
\hline Lejeunea flava (Sw.) Nees & + & + & & & Pantropical & $\begin{array}{l}\text { AC, AM, BA, ES, GO, } \\
\text { MG, PA, PE, RR, RS, SP. }\end{array}$ \\
\hline $\begin{array}{l}\text { Lejeunea grossiretis (Steph.) E. Reiner } \\
\text { \& Goda }\end{array}$ & & & + & & Neotropical & BA, RJ. \\
\hline $\begin{array}{l}\text { Lejeunea huctumalcensis Lindenb. } \\
\text { \& Gottsche }\end{array}$ & + & & + & + & Neotropical & AL, AM, BA, PA, SP. \\
\hline Lejeunea immersa Spruce & + & + & & & Neotropical & BA. \\
\hline Lejeunea laetevirens Nees \& Mont. & + & & & & Neotropical & BA, ES, PA, PE, RJ, SP. \\
\hline Lejeunea oligoclada Spruce & + & + & + & + & Neotropical & BA, ES, MG, RJ, SC, SP. \\
\hline Lejeunea perpapillosa M.E. Reiner \& K.C. Pôrto & + & + & + & + & Neotropical & BA, PE. \\
\hline Lejeunea phyllobola Nees \& Mont. & + & + & & + & Neotropical & $\begin{array}{c}\mathrm{AC}, \mathrm{AM}, \mathrm{BA}, \mathrm{ES}, \mathrm{PA}, \mathrm{RJ}, \\
\text { RS, SP. }\end{array}$ \\
\hline Lejeunea tapajosensis Spruce & + & + & + & & Neotropical & $\mathrm{AC}, \mathrm{BA}, \mathrm{ES}, \mathrm{PA}$. \\
\hline Lepidolejeunea involuta (Gottsche) Grolle & & & & + & Neotropical & $\begin{array}{c}\text { AM, BA, ES, PA, PR, RO, } \\
\text { RR, SP. }\end{array}$ \\
\hline $\begin{array}{l}\text { Leptolejeunea elliptica (Lehm. \& Lindenb.) } \\
\text { Schiffn. }\end{array}$ & + & & + & + & Neotropical & $\begin{array}{c}\text { AC, AL, AM, AP, BA, ES, } \\
\text { MG, PA, PR, RJ, RR, SC, } \\
\text { SP. }\end{array}$ \\
\hline Leptolejeunea exocellata (Spruce) A. Evans & + & & & & Neotropical & $\begin{array}{l}\text { AL, BA, MS, MT, PR, } \\
\text { RJ, SP. }\end{array}$ \\
\hline Leptolejeunea moniliata Steph. & + & & & & Neotropical & AM, BA, RJ. \\
\hline Lopholejeunea nigricans (Lindenb.) Schiffn. & + & & + & + & Pantropical & $\begin{array}{l}\text { AM, BA, GO, MG, MT, } \\
\quad \text { PA, PR, RJ, SP. }\end{array}$ \\
\hline Lopholejeunea subfusca (Nees) Schiffn. & + & + & + & + & Pantropical & $\begin{array}{l}\text { AC, AM, BA, ES, MT, } \\
\text { PA, PB, PE, RJ, RO, RR, } \\
\text { SC, SP. }\end{array}$ \\
\hline Microlejeunea acutifolia Steph. & & + & & & Neotropical & $\mathrm{AM}, \mathrm{BA}, \mathrm{PA}$. \\
\hline Microlejeunea bullata (Taylor) Steph. & + & & & & Neotropical & $\begin{array}{l}\text { AC, BA, ES, RJ, RR, } \\
\text { SE, SP. }\end{array}$ \\
\hline Microlejeunea epiphylla Bischl. & + & + & & + & Neotropical & $\mathrm{BA}, \mathrm{PE}, \mathrm{PR}, \mathrm{SP}$. \\
\hline $\begin{array}{l}\text { Microlejeunea stricta (Lindenb. \& Gottsche) } \\
\text { Steph. }\end{array}$ & + & & & & Neotropical & BA, MG, PA, SP. \\
\hline Prionolejeunea aemula (Gottsche) A. Evans & + & & & & Neotropical & $\mathrm{BA}, \mathrm{RJ}, \mathrm{SE}, \mathrm{SP}$. \\
\hline Prionolejeunea denticulata (F. Weber) Schiffn. & + & + & + & + & Neotropical & AM, BA, PA, RJ. \\
\hline Prionolejeunea grata (Gottsche) Schiffn. & + & & + & + & Neotropical & $\begin{array}{l}\text { BA, CE, GO, ES, MS, } \\
\text { MT, PB, RJ, RS, SP. }\end{array}$ \\
\hline Prionolejeunea scaberula (Spruce) Steph. & + & + & + & + & Neotropical & BA. \\
\hline
\end{tabular}


Tabela 1 (continuação)

\begin{tabular}{|c|c|c|c|c|c|c|}
\hline Táxons & MV & PG & LI & PAC & $\begin{array}{l}\text { Distribuição } \\
\text { mundial }\end{array}$ & Distribuição no Brasil \\
\hline Pycnolejeunea contigua (Nees) Grolle & & + & & + & Pantropical & $\begin{array}{l}\text { AM, BA, ES, MG, PA, } \\
\text { PE, RR, RS, SC, SP. }\end{array}$ \\
\hline $\begin{array}{l}\text { Pycnolejeunea densistipula (Lehm. } \\
\text { \& Lindenb.) Steph. }\end{array}$ & & + & & + & Neotropical & BA, RJ, SC, SP. \\
\hline Pycnolejeunea macroloba (Nees \& Mont.) Schiffn. & & + & + & + & Neotropical & AM, BA, PA, PE, SP. \\
\hline $\begin{array}{l}\text { Rectolejeunea berteroana (Gottsche ex Steph.) } \\
\text { A. Evans }\end{array}$ & + & + & + & & Neotropical & AC, AM, BA, ES, PR, SP. \\
\hline $\begin{array}{l}\text { Rectolejeunea emarginuliflora (Gottsche ex } \\
\text { Steph.) A. Evans }\end{array}$ & & + & + & & Neotropical & BA, ES, SP. \\
\hline Rectolejeunea flagelliformis A. Evans & & & + & & Neotropical & BA, PE. \\
\hline Rectolejeunea truncatilobula C. Bastos & + & & + & + & Neotropical & BA, SP. \\
\hline Schiffneriolejeunea polycarpa (Nees) Gradst. & & & + & & Pantropical & $\begin{array}{l}\text { AM, BA, ES, GO, MG, } \\
\text { PA, PE, RJ, RS, SC, SP. }\end{array}$ \\
\hline Stictolejeunea squamata (Willd. ex Web.) Schiffn. & + & + & + & + & Neotropical & $\begin{array}{l}\mathrm{AC}, \mathrm{AM}, \mathrm{BA}, \mathrm{MG}, \mathrm{PA} \\
\text { PE, RJ, SP. }\end{array}$ \\
\hline $\begin{array}{l}\text { Symbiezidium barbiflorum (Lindenb. \& } \\
\text { Gottsche) A. Evans }\end{array}$ & + & + & + & + & Pantropical & $\begin{array}{l}\text { AM, BA, ES, PA, PE, RJ, } \\
\text { SC, SP. }\end{array}$ \\
\hline $\begin{array}{l}\text { Symbiezidium transversale var. hookerianum ( } \\
\text { Gottsche et al.) Gradst. \& Van Beek }\end{array}$ & + & + & + & + & Neotropical & $\begin{array}{l}\text { AM, AP, BA, CE, ES, PA, } \\
\text { RJ, SP. }\end{array}$ \\
\hline $\begin{array}{l}\text { Symbiezidium transversale (Sw.) Trevis } \\
\text { var. transversale }\end{array}$ & & & + & & Neotropical & AP, BA. \\
\hline Taxilejeunea obtusangula (Spruce) A. Evans & + & & + & & Neotropical & BA, PA, PR, RR, SP. \\
\hline $\begin{array}{l}\text { Taxilejeunea pterigonia (Lehm. \& Lindenb.) } \\
\text { Schiffn. }\end{array}$ & & + & & + & Neotropical & BA, ES, MG, RJ, SC, SP. \\
\hline $\begin{array}{l}\text { Vitalianthus bischlerianus (K.C. Pôrto \& Grolle) } \\
\text { R.M. Schust. \& Giancottti }\end{array}$ & & + & + & + & Neotropical & $\begin{array}{l}\mathrm{BA}, \mathrm{ES}, \mathrm{PE}, \mathrm{PR}, \mathrm{RJ}, \mathrm{SC} \\
\text { SP. }\end{array}$ \\
\hline $\begin{array}{l}\text { Xylolejeunea crenata (Nees \& Mont.) X.-L. } \\
\text { He \& Grolle }\end{array}$ & & & + & & Neotropical & $\begin{array}{l}\mathrm{AM}, \mathrm{BA}, \mathrm{MA}, \mathrm{MG}, \mathrm{PA} \\
\text { PE, RO, SP. }\end{array}$ \\
\hline \multicolumn{7}{|l|}{ LEPIDOZIACEAE } \\
\hline Micropterygium campanense Spruce ex Reimers & & + & & + & Neotropical & BA, MG. \\
\hline Micropterygium reimersianum Herzog & & & & + & Neotropical & BA. \\
\hline Micropterygium trachyphyllum Reimers & & + & & & Neotropical & $\mathrm{BA}, \mathrm{PA}, \mathrm{RR}$. \\
\hline $\begin{array}{l}\text { Telaranea nematodes (Gottsche ex Austin) } \\
\text { M.A. Howe }\end{array}$ & & + & & & Pantropical & $\begin{array}{l}\text { AC, AM, BA, ES, MG, } \\
\text { RJ, RR, SC, SP. }\end{array}$ \\
\hline \multicolumn{7}{|l|}{ LOPHOCOLEACEAE } \\
\hline $\begin{array}{l}\text { Chiloscyphus martianus (Nees) J.J. Engel \& } \\
\text { R.M. Schust. }\end{array}$ & & + & & + & Pantropical & $\begin{array}{l}\text { AM, AP, BA, ES, MG, PA, } \\
\text { PE, PR, RS, SC, } \\
\text { SE, SP. }\end{array}$ \\
\hline $\begin{array}{l}\text { Chiloscyphus muricatus (Lehm.) J.J. Engel \& } \\
\text { R.M. Schust. }\end{array}$ & & & & + & Neotropical & BA, ES, MG, RJ, RS, SP. \\
\hline $\begin{array}{l}\text { Chiloscyphus perissodontus (Lehm.) Steph. \& } \\
\text { R.M. Schust. }\end{array}$ & & & & + & Neotropical & $\begin{array}{l}\mathrm{AM}, \mathrm{AP}, \mathrm{BA}, \mathrm{MG}, \mathrm{PA} \\
\text { RJ, SC, SP. }\end{array}$ \\
\hline
\end{tabular}


Tabela 1 (continuação)

\begin{tabular}{|c|c|c|c|c|c|c|}
\hline Táxons & MV & PG & LI & PAC & $\begin{array}{l}\text { Distribuição } \\
\text { mundial }\end{array}$ & Distribuição no Brasil \\
\hline $\begin{array}{l}\text { *Chiloscyphus proteus (Herzog) J.J. Engel \& R.M. } \\
\text { Schust. }\end{array}$ & & & + & & Neotropical & SP, RJ. \\
\hline $\begin{array}{l}\text { *Chiloscyphus serratus (Mitt.) J.J. Engel \& R.M. } \\
\text { Schust. }\end{array}$ & & & & + & Neotropical & CE, MT. \\
\hline METZGERIACEAE & & & & & Neotropical & \\
\hline Metzgeria aurantiaca Steph. & + & & & & Neotropical & $\begin{array}{l}\text { AM, BA, CE, ES, PB, PE, } \\
\text { PR, RJ, RS, SC, SP. }\end{array}$ \\
\hline Metzgeria brasiliensis Schiffn. & & & + & + & Neotropical & BA, PR, SC, SP, RJ, RS. \\
\hline Metzgeria furcata (L.) Dumort. & + & + & & + & Neotropical & $\begin{array}{c}\mathrm{AC}, \mathrm{BA}, \mathrm{CE}, \mathrm{ES}, \mathrm{GO}, \\
\mathrm{MG}, \mathrm{PB}, \mathrm{PE}, \mathrm{RJ}, \mathrm{RS}, \\
\text { SC, SP. }\end{array}$ \\
\hline \multicolumn{7}{|l|}{ PLAGIOCHILACEAE } \\
\hline Plagiochila adiantoides (Sw.) Lindenb. & + & & + & + & Neotropical & BA, GO, RS, RJ. \\
\hline Plagiochila aerea Taylor & + & & + & + & Neotropical & BA, PA. \\
\hline Plagiochila bifaria (Sw.) Lindenb. & + & & & & Neotropical & BA, PR, RJ. \\
\hline Plagiochila corrugata (Nees) Nees \& Mont. & + & & & + & Neotropical & $\begin{array}{l}\text { BA, CE, DF, GO, MG, RJ, } \\
\text { RS, SC, SP. }\end{array}$ \\
\hline $\begin{array}{l}\text { Plagiochila disticha (Lehm. \& Lindenb.) } \\
\text { Lindenb. }\end{array}$ & + & + & + & + & Neotropical & $\begin{array}{c}\text { AC, AM, AP, BA, CE, } \\
\text { MT, PA, PB, PE, RJ, RR, } \\
\text { RS, SP. }\end{array}$ \\
\hline $\begin{array}{l}\text { Plagiochila gymnocalycina (Lehm. \& } \\
\text { Lindenb.) Lindenb. }\end{array}$ & + & + & + & + & Neotropical & BA, MG, PE, RJ, RS, SP. \\
\hline Plagiochila montagnei Nees & + & + & & + & Neotropical & $\begin{array}{l}\text { AM, AP, BA, PA, PE, PR, } \\
\text { RJ, SP. }\end{array}$ \\
\hline Plagiochila patentissima Lindenb. & + & & + & + & Neotropical & $\begin{array}{l}\text { BA, ES, MG, PE, PR, RJ, } \\
\text { RS, SC, SP. }\end{array}$ \\
\hline Plagiochila patula (Sw.) Lindenb. & + & & + & & Neotropical & BA, RJ. \\
\hline Plagiochila raddiana Lindenb. & + & & + & & Neotropical & $\begin{array}{l}\text { BA, ES, MG, MT, PA, PE, } \\
\text { PR, SP. }\end{array}$ \\
\hline Plagiochila rutilans Lindenb. var. rutilans & + & & & & Neotropical & $\begin{array}{l}\text { AM, AP, BA, MG, PA, PE, } \\
\text { RJ, RR, SC, SP. }\end{array}$ \\
\hline $\begin{array}{l}\text { Plagiochila rutilans var. moritziana (Gottsche } \\
\text { \& Lindenb. ex Hampe) J. Heinrichs }\end{array}$ & + & & & & Neotropical & $\begin{array}{l}\text { AC, AM, AP, BA, RJ, } \\
\text { RS, SC. }\end{array}$ \\
\hline Plagiochila simplex (Sw.) Lindenb. & + & + & + & + & Neotropical & $\begin{array}{l}\text { AM, BA, ES, MG, PE, } \\
\text { PR, RJ, RS, SP. }\end{array}$ \\
\hline Plagiochila subplana Lindenb. & + & & + & & Neotropical & $\begin{array}{l}\text { AM, BA, MG, PA, RJ, } \\
\text { SC, SP. }\end{array}$ \\
\hline \multicolumn{7}{|l|}{ RADULACEAE } \\
\hline Radula flaccida Lindenb. \& Gottsche & & + & & & Pantropical & BA, PA, RR, SP. \\
\hline Radula javanica Gottsche & + & + & + & + & Pantropical & BA, PA, RR, SP. \\
\hline Radula kegelii Gottsche ex Steph. & & & + & & Neotropical & $\begin{array}{l}\text { BA, MT, PA, PR, RJ, RS, } \\
\text { SC, SP. }\end{array}$ \\
\hline
\end{tabular}


Tabela 1 (continuação)

\begin{tabular}{|c|c|c|c|c|c|c|}
\hline Táxons & MV & PG & LI & PAC & $\begin{array}{l}\text { Distribuição } \\
\text { mundial }\end{array}$ & Distribuição no Brasil \\
\hline Radula ligula Steph. & & & + & & Neotropical & $\mathrm{AL}, \mathrm{BA}, \mathrm{RJ}, \mathrm{RS}, \mathrm{SC}, \mathrm{SP}$. \\
\hline Radula mammosa Spruce & + & & & & Neotropical & AM, BA, SP. \\
\hline Radula mexicana Lindenb. \& Gottsche & + & + & & & Neotropical & BA, ES, RJ, RS, SP. \\
\hline Radula recubans Taylor & + & & + & + & Neotropical & BA, PA, RJ, RS, SP. \\
\hline \multicolumn{7}{|l|}{ BRYOPHYTA } \\
\hline \multicolumn{7}{|l|}{ BRACHYTHECIACEAE } \\
\hline Helicodontium capillare (Hedw.) A. Jaeger & & & & + & Neotropical & $\begin{array}{l}\mathrm{BA}, \mathrm{MG}, \mathrm{PR}, \mathrm{RJ}, \mathrm{RS} \\
\text { SC, SP. }\end{array}$ \\
\hline Zelometeorium patulum (Hedw.) Manuel & + & + & + & & Neotropical & $\begin{array}{l}\text { AC, BA, AM, GO, MG, } \\
\text { MT, PA, PR, RJ, SC, SP. }\end{array}$ \\
\hline Zelometeorium recurvifolium (Hornsch.) Manuel & + & & + & & Neotropical & $\begin{array}{c}\text { AL, AM, BA, ES, MS, } \\
\text { MT, PA, PR, RJ, RR, RS, } \\
\text { RO, SC, SP. }\end{array}$ \\
\hline \multicolumn{7}{|l|}{ BRYACEAE } \\
\hline Rosulabryum billarderi (Schwagr.) J.R. Spence & & & + & & Pantropical & $\begin{array}{c}\text { AM, BA, ES, GO, MA, } \\
\text { MG, MT, PA, RJ, RO, } \\
\text { SC, SP. }\end{array}$ \\
\hline \multicolumn{7}{|l|}{ CALYMPERACEAE } \\
\hline Calymperes afzelii Sw. & & + & & & Neotropical & $\begin{array}{l}\text { AC, AM, BA, MS, MT, } \\
\text { PA, PB, PE, RJ, RO, RR, } \\
\text { SC, SP, TO. }\end{array}$ \\
\hline Calymperes erosum Müll. Hal. & & + & + & + & Neotropical & $\begin{array}{c}\text { AC, AP, MA, BA, MG, } \\
\text { MT, PA, PB, PE, RJ, RO, } \\
\text { RR. }\end{array}$ \\
\hline *Calymperes levyanum Besch. & + & & + & & Neotropical & AM, MT, ES, PA. \\
\hline Calymperes lonchophyllum Schwägr. & + & + & + & + & Neotropical & $\begin{array}{c}\text { AC, AL, AM, AP, BA, ES, } \\
\text { MT, PA, PE, PR, RJ, RO, } \\
\text { RR, SP. }\end{array}$ \\
\hline Calymperes nicaraguense Renauld \& Cardot & + & & & & Neotropical & $\begin{array}{l}\mathrm{AM}, \mathrm{AP}, \mathrm{BA}, \mathrm{MT} \\
\text { PA, RO. }\end{array}$ \\
\hline Calymperes palisotii Schwagr. & & + & & + & Neotropical & $\begin{array}{c}\text { AL, AM, AP, BA, CE, ES, } \\
\text { FN, PA, PB, PE, PI, PR, } \\
\text { RJ, RN, RO, RR e SE. }\end{array}$ \\
\hline Calymperes rubiginosum (Mitt.) Reese & & + & & & Neotropical & AM, BA, PA, RO, RR. \\
\hline *Calymperes tenerum Müll. Hal. & & & + & & Pantropical & RJ, SP. \\
\hline Leucophanes molleri Müll. Hal. & & & & + & Neotropical & AM, BA, PA, RO, RR, SP. \\
\hline Octoblepharum albidum Hedw. & + & + & + & & Pantropical & $\begin{array}{c}\text { AC, AL, AM, AP, BA, ES, } \\
\text { GO, MT, MS, PA, PE, PB, } \\
\text { PI, PR, RJ, RO, RS, RR, } \\
\text { SC, SE, SP, TO. }\end{array}$ \\
\hline $\begin{array}{l}\text { Octoblepharum pulvinatum (Dozy \& Molk.) } \\
\text { Mitt. }\end{array}$ & + & + & & + & Pantropical & $\begin{array}{c}\text { AC, AM, AP, BA, ES, } \\
\text { MA, MS, MT, PA, PE, RJ, } \\
\text { RO, RR, SP. }\end{array}$ \\
\hline
\end{tabular}


Tabela 1 (continuação)

\begin{tabular}{|c|c|c|c|c|c|c|}
\hline Táxons & MV & PG & LI & PAC & $\begin{array}{l}\text { Distribuição } \\
\text { mundial }\end{array}$ & Distribuição no Brasil \\
\hline Syrrhopodon gardneri (Hook.) Schwägr. & + & & & & Neotropical & $\begin{array}{l}\text { AM, BA, GO, MT, } \\
\text { MG, RJ. }\end{array}$ \\
\hline Syrrhopodon gaudichaudii Mont. & + & + & + & + & Pantropical & $\begin{array}{l}\text { AM, BA, GO, MA, MG, } \\
\text { MT, PA, PE, PR, RJ, RS, } \\
\text { SC, SP. }\end{array}$ \\
\hline $\begin{array}{l}\text { Syrrhopodon incompletus Schwägr. var. } \\
\text { incompletus }\end{array}$ & + & & & & Pantropical & $\begin{array}{l}\text { AC, AM, AP, BA, GO, } \\
\text { MG, MS, MT, PA, PE, PR, } \\
\text { RJ, RO, RR, SC, SP. }\end{array}$ \\
\hline $\begin{array}{l}\text { Syrrhopodon incompletus var. berteroanus } \\
\text { (Brid.) W.D. Reese }\end{array}$ & + & & & & Neotropical & AP, BA, PR, RJ, SC, SP. \\
\hline $\begin{array}{l}\text { Syrrhopodon incompletus var. lanceolatus } \\
\text { (Hampe) W.D. Reese }\end{array}$ & & & & + & Neotropical & $\begin{array}{l}\text { AM, BA, PA, PB, PR, RJ, } \\
\text { SC, SP. }\end{array}$ \\
\hline Syrrhopodon ligulatus Mont. & & + & + & & Pantropical & $\begin{array}{c}\text { AM, AP, BA, GO, PA, PE, } \\
\text { MG, MS, MT, RJ, RO, } \\
\text { RR, SP. }\end{array}$ \\
\hline Syrrhopodon parasiticus (Brid.) Besch. & & + & & + & Pantropical & $\begin{array}{l}\text { AC, AM, BA, ES, GO, } \\
\text { MG, MS, MT, PA, PE, PR, } \\
\text { RJ, RO, RR, SC, SP. }\end{array}$ \\
\hline Syrrhopodon prolifer Schwägr. var. prolifer & & + & + & & Pantropical & $\begin{array}{c}\text { AL, AM, BA, ES, GO, M } \\
\text { G, MT, PA, PE, PI, PR, } \\
\text { RJ, RO, RS, SC, SE, } \\
\text { SP, TO. }\end{array}$ \\
\hline $\begin{array}{l}\text { Syrrhopodon prolifer (Mitt.) W.D. Reese var. } \\
\text { scaber }\end{array}$ & & + & & + & Pantropical & $\begin{array}{l}\text { AC, AM, BA, MG, PA, } \\
\text { PR, RJ, RO, SC, SP. }\end{array}$ \\
\hline \multicolumn{7}{|l|}{ DICRANACEAE } \\
\hline Holomitrium crispulum Mart. & & & & + & Neotropical & BA, DF, GO, RJ, RS, SP. \\
\hline Leucoloma serrulatum Brid. & + & + & & & Neotropical & $\begin{array}{l}\text { AL, BA, ES, MG, PE, } \\
\text { RJ, SP. }\end{array}$ \\
\hline \multicolumn{7}{|l|}{ FISSIDENTACEAE } \\
\hline Fissidens asplenioides Hedw. & & & & + & Neotropical & $\begin{array}{l}\text { BA, CE, MG, MT, PR, RJ, } \\
\text { RS, RR, SC, SP. }\end{array}$ \\
\hline Fissidens pellucidus Hornsch. & + & + & + & + & Neotropical & $\begin{array}{l}\text { AM, BA, PA, RO, RR, } \\
\text { SC, SP. }\end{array}$ \\
\hline Fissidens santa-clarensis Thér. & + & + & + & & Neotropical & $\mathrm{BA}, \mathrm{GO}, \mathrm{PA}$. \\
\hline Fissidens scariosus Mitt. & + & + & & + & Neotropical & $\begin{array}{l}\text { BA, ES, MG, PA, PE, PR, } \\
\text { RJ, RO, RR, SC, SP. }\end{array}$ \\
\hline Fissidens zollingeri Mont. & + & & & & Neotropical & $\begin{array}{c}\text { AC, AM, BA, ES, GO, } \\
\text { MA, MG, MS, MT, PA, } \\
\text { PB, PI, PR, RJ, RO, RR, } \\
\text { RS, SC, TO. }\end{array}$ \\
\hline \multicolumn{7}{|l|}{ HYPNACEAE } \\
\hline Chryso-hypnum diminutivum (Hampe) W.R. Buck & + & & & & Neotropical & $\begin{array}{c}\text { AC, AM, AP, BA, DF, ES, } \\
\text { GO, MG, MS, MT, PA, PE, } \\
\text { PR, RJ, RR, RS, SC, SP. }\end{array}$ \\
\hline
\end{tabular}


Tabela 1 (continuação)

\begin{tabular}{|c|c|c|c|c|c|c|}
\hline Táxons & MV & PG & LI & PAC & $\begin{array}{l}\text { Distribuição } \\
\text { mundial }\end{array}$ & Distribuição no Brasil \\
\hline Chryso-hypnum elegantulum (Hook.) Hampe & + & & & & Neotropical & $\begin{array}{c}\text { AM, BA, DF, ES, GO, } \\
\text { MG, MS, MT, PR, RJ, RS, } \\
\text { SC, SP. }\end{array}$ \\
\hline $\begin{array}{l}\text { Ectropothecium leptochaeton (Schwägr.) W.R. } \\
\text { Buck }\end{array}$ & + & & & + & Neotropical & $\begin{array}{l}\text { AM, BA, ES, MG, MS, } \\
\text { MT, PA, PR, RJ, SC. }\end{array}$ \\
\hline Vesicularia vesicularis (Schwägr.) Broth. & + & & & + & Neotropical & $\begin{array}{l}\text { AC, AL, AM, BA, MG, } \\
\text { MS, MT, PA,PE, PI, RJ, } \\
\text { RS, RR, RO, SC, SP. }\end{array}$ \\
\hline \multicolumn{7}{|l|}{ LEUCOBRYACEAE } \\
\hline Leucobryum giganteum Müll. Hal. & & + & & & Neotropical & $\begin{array}{l}\text { AM, BA, ES, MG, PE, } \\
\text { PR, RJ, SC, SP. }\end{array}$ \\
\hline Leucobryum martianum (Hornsch.) Hampe & & + & & + & Pantropical & $\begin{array}{l}\mathrm{AC}, \mathrm{AL}, \mathrm{AM}, \mathrm{AP}, \mathrm{BA}, \mathrm{CE}, \\
\mathrm{ES}, \mathrm{GO}, \mathrm{MA}, \mathrm{MG}, \mathrm{MS}, \\
\mathrm{MT}, \mathrm{PA}, \mathrm{PE}, \mathrm{PR}, \mathrm{RJ}, \mathrm{RO}, \\
\text { RR, RS, SC, SE, SP. }\end{array}$ \\
\hline Ochrobryum gardneri (Müll. Hal.) Mitt. & & + & & + & Pantropical & $\begin{array}{l}\text { AL, BA, CE, DF, ES, GO, } \\
\text { MG, MS, MT, PA, PE, RJ, } \\
\text { RO, RR, SP, TO }\end{array}$ \\
\hline \multicolumn{7}{|l|}{ METEORIACEAE } \\
\hline Meteoridium remotifolium (Mull. Hal.) Manuel & + & + & & & Neotropical & $\begin{array}{l}\text { AL, BA, ES, RJ, RR, RS, } \\
\text { RR, SC, SP. }\end{array}$ \\
\hline Pseudotrachypus martinicensis (Broth.) W.R. Buck & & + & & & Neotropical & BA. \\
\hline Squamidium leucotrichum (Taylor) Broth. & + & & & & Neotropical & $\begin{array}{l}\mathrm{AC}, \mathrm{AM}, \mathrm{BA}, \mathrm{CE}, \mathrm{ES}, \mathrm{PA}, \\
\quad \text { PE, RJ, RR, RS, SP. }\end{array}$ \\
\hline Squamidium nigricans (Hook.) Broth. & + & & & & Neotropical & $\begin{array}{l}\mathrm{CE}, \mathrm{GO}, \mathrm{MG}, \mathrm{PR}, \mathrm{RJ}, \mathrm{RS}, \\
\text { SP. }\end{array}$ \\
\hline \multicolumn{7}{|l|}{ NECKERACEAE } \\
\hline Homalia glabella (Hedw.) Schimp. & + & & & & Neotropical & $\mathrm{BA}, \mathrm{RJ}, \mathrm{SC}, \mathrm{SP}$. \\
\hline Homaliodendron piniforme (Brid.) Enroth & + & & & & Pantropical & BA, PE, RJ, SP. \\
\hline Isodrepanium lentulum (Wilson) E. Britton & & + & & & Neotropical & BA, RJ, RO, SP. \\
\hline Neckeropsis disticha (Hedw.) Kindb. & + & & & + & Pantropical & $\begin{array}{l}\text { AC, AM, BA, ES, MG, } \\
\text { PA, PR, RJ, RR, SC, SP. }\end{array}$ \\
\hline Neckeropsis undulata (Hedw.) Reichardt & + & + & & + & Neotropical & $\begin{array}{c}\text { AC, AL, AM, AP, BA, } \\
\text { CE, DF, ES, GO, MT, MS, } \\
\text { MG, PA, PE, PR. }\end{array}$ \\
\hline Porotrichum substriatum (Hampe) Mitt. & & + & + & & Pantropical & $\begin{array}{l}\text { AL, BA, PA, PE, RJ, RO, } \\
\text { RS, SC, SP. }\end{array}$ \\
\hline \multicolumn{7}{|l|}{ ORTHOTRICHACEAE } \\
\hline Groutiella apiculata (Hook.) H.A. Crum \& Steere & + & + & + & + & Neotropical & $\begin{array}{l}\text { BA, ES, MS, MT, PA, PE, } \\
\text { PR, RJ, SP. }\end{array}$ \\
\hline *Groutiella obtusa (Mitt.) Florsch. & + & + & + & & Neotropical & PA, RO. \\
\hline Macromitrium cirrosum (Hedw.) Brid. & + & & & & Neotropical & $\begin{array}{l}\text { AP, BA, CE, ES, PA, PE, } \\
\quad \text { PR, RJ, RS, SC, SP. }\end{array}$ \\
\hline
\end{tabular}


Tabela 1 (continuação)

\begin{tabular}{|c|c|c|c|c|c|c|}
\hline Táxons & MV & PG & $\mathrm{LI}$ & PAC & $\begin{array}{l}\text { Distribuição } \\
\text { mundial }\end{array}$ & Distribuição no Brasil \\
\hline Macromitrium richardii Schwägr. & + & & & & Neotropical & $\begin{array}{c}\text { AL, AM, BA, CE, ES, } \\
\text { MG, PE, PR, RJ, RS, SC, } \\
\text { SP. }\end{array}$ \\
\hline Schlotheimia rugifolia (Hook.) Schwagr. & + & & + & + & Neotropical & $\begin{array}{l}\text { AC, AL, AM, BA, CE, } \\
\text { DF, ES, GO, MG, MT, PA, } \\
\text { PE, PR, RJ, RO, RS, SC, } \\
\text { SP, TO. }\end{array}$ \\
\hline \multicolumn{7}{|l|}{ PHYLLOGONIACEAE } \\
\hline Phyllogonium fulgens (Hedw.) Brid. & + & & & & Neotropical & BA, MG, PA, PE, RJ, SC. \\
\hline Phyllogonium viride Brid. & + & & & & Neotropical & $\begin{array}{l}\text { AL, BA, CE, ES, MG, PE, } \\
\quad \text { PR, RJ, RS, SC, SP. }\end{array}$ \\
\hline \multicolumn{7}{|l|}{ PILOTRICHACEAE } \\
\hline *Callicostella merkelii (Hornsch.) A. Jaeger & & & + & & Neotropical & $\begin{array}{c}\text { AC, AM, AP, CE, GO, } \\
\text { MG, PA, PE, RJ, RR, SC, } \\
\text { SP. }\end{array}$ \\
\hline Callicostella pallida (Hornsch.) Angstr. & + & & & & Neotropical & $\begin{array}{c}\mathrm{AC}, \mathrm{AM}, \mathrm{AP}, \mathrm{BA}, \mathrm{CE}, \mathrm{ES}, \\
\mathrm{GO}, \mathrm{MG}, \mathrm{MS}, \mathrm{MT}, \mathrm{PA}, \\
\mathrm{PE}, \mathrm{PR}, \mathrm{RJ}, \mathrm{RO}, \mathrm{RR}, \mathrm{RS}, \\
\text { SE e SP. }\end{array}$ \\
\hline Lepidopilum scabrisetum (Schwagr.) Steere & & & + & + & Neotropical & $\begin{array}{c}\text { AM, AP, BA, CE, MG, } \\
\text { PA, PE, RJ, RR, RS, SC, } \\
\text { SP. }\end{array}$ \\
\hline Lepidopilum surinamense Müll. Hal. & & & + & & Neotropical & $\begin{array}{c}\mathrm{AL}, \mathrm{AM}, \mathrm{AP}, \mathrm{BA}, \mathrm{PA}, \mathrm{RJ} \\
\mathrm{RO}, \mathrm{SP} .\end{array}$ \\
\hline Pilotrichum bipinnatum (Schwägr.) Brid. & & + & & + & Neotropical & $\begin{array}{l}\text { AC, AM, AP, BA, MA, } \\
\text { MG, PA, RJ, RO, RR, SP. }\end{array}$ \\
\hline Pilotrichum evanescens (Müll. Hal.) Crosby & & + & & + & Neotropical & $\begin{array}{l}\text { AM, BA, MG, MT, PA, } \\
\text { PE, PR, RJ, RR, SC, SP. }\end{array}$ \\
\hline \multicolumn{7}{|l|}{ PTEROBRYACEAE } \\
\hline Henicodium geniculatum (Mitt.) W.R. Buck & & + & + & + & Neotropical & $\begin{array}{c}\text { AC, AL, AM, AP, BA, CE, } \\
\text { DF, ES, GO, MT, PA, PE, } \\
\text { RJ, RO, RS, SP. }\end{array}$ \\
\hline Orthostichopsis praetermissa W.R. Buck & + & & & & Neotropical & AM, BA. \\
\hline \multicolumn{7}{|l|}{ PYLAISIADELPHACEAE } \\
\hline Isopterygium tenerifolium Mitt. & & & + & + & Neotropical & $\begin{array}{c}\mathrm{AC}, \mathrm{AL}, \mathrm{AM}, \mathrm{AP}, \mathrm{BA}, \mathrm{CE}, \\
\mathrm{DF}, \mathrm{ES}, \mathrm{GO}, \mathrm{MT}, \mathrm{PA}, \mathrm{PB}, \\
\text { PE, RJ, RO, SP. }\end{array}$ \\
\hline Isopterygium tenerum (Sw.) Mitt. & + & & & & Neotropical & $\begin{array}{l}\text { AC, AM, BA, DF, ES, } \\
\text { GO, MG, MS, MT, PA, } \\
\text { PB, PE, PI, PR, RJ, RR, } \\
\text { RS, RO, SC, SP, TO. }\end{array}$ \\
\hline $\begin{array}{l}\text { Pterogonidium pulchellum (Hook.) Müll. Hal. } \\
\text { ex Broth. }\end{array}$ & & & + & & Neotropical & $\begin{array}{l}\text { AM, BA, CE, PA, PE, PR, } \\
\text { RJ, RS, RO, SP. }\end{array}$ \\
\hline
\end{tabular}


Tabela 1 (continuação)

\begin{tabular}{|c|c|c|c|c|c|c|}
\hline Táxons & MV & PG & LI & PAC & $\begin{array}{l}\text { Distribuição } \\
\text { mundial }\end{array}$ & Distribuição no Brasil \\
\hline Taxithelium planum (Brid.) Mitt. & + & + & + & + & Pantropical & $\begin{array}{c}\text { AC, AL, AM, BA, ES, } \\
\text { GO, MA, MG, MS, MT, } \\
\text { PA, PB, PR, RJ, RO, RR, } \\
\text { SC, SP, TO. }\end{array}$ \\
\hline $\begin{array}{l}\text { Taxithelium pluripunctatum (Renauld \& Cardot) } \\
\text { W.R. Buck }\end{array}$ & + & + & + & + & Neotropical & AM, BA, ES, PA, PE, RR. \\
\hline Taxithelium portoricense R.S. Williams & & + & + & + & Neotropical & BA, PA. \\
\hline \multicolumn{7}{|l|}{ SEMATOPHYLLACEAE } \\
\hline Acroporium pungens (Hedw.) Broth. & & + & + & + & Pantropical & $\begin{array}{c}\text { AM, AP, BA, CE, DF, ES, } \\
\text { GO, MG, PA, PR, RJ, RO, } \\
\text { RR, SC, SP. }\end{array}$ \\
\hline Sematophyllum adnatum (Michx.) E. Britton & & + & + & + & Pantropical & $\begin{array}{l}\text { AM, BA, ES, MT, PA, RJ, } \\
\text { RS, SC, SP, TO. }\end{array}$ \\
\hline Sematophyllum beyrichii (Hornsch.) Broth. & & + & + & + & Neotropical & $\begin{array}{l}\text { BA, ES, PE, PR, RJ, RS, } \\
\text { SC, SP. }\end{array}$ \\
\hline Sematophyllum galipense (Müll. Hal.) Mitt. & + & & & & Neotropical & $\begin{array}{l}\text { BA, CE, ES, GO, MG, } \\
\text { MT, PA, PE, PR, RJ, RO, } \\
\text { RR, RS, SC e SP. }\end{array}$ \\
\hline Sematophyllum subpinnatum (Brid.) E. Britton & & + & + & & Pantropical & $\begin{array}{c}\text { AC, AL, AM, AP, BA, CE, } \\
\text { DF, ES, GO, MG, MS, } \\
\text { MT, PA, PB, PE, PR, RJ, } \\
\text { RO, RS, RR, SC, SP, TO }\end{array}$ \\
\hline Sematophyllum subsimplex (Hedw.) Mitt. & + & + & + & + & Pantropical & $\begin{array}{l}\text { AC, AM, AP, BA, CE, DF, } \\
\text { ES, GO MA, MG, MS, } \\
\text { MT, PA, PB, PE, PI, PR, } \\
\text { RJ, RO, RS, RR, SC, SE, } \\
\text { SP, TO. }\end{array}$ \\
\hline Sematophyllum tequendamense (Hampe) Mitt. & & + & + & + & Neotropical & BA. \\
\hline Trichosteleum brachydictyon (Besch.) A. Jaeger & + & + & & & Neotropical & BA. \\
\hline $\begin{array}{l}\text { Trichosteleum microstegium (Schimp. ex Besch.) } \\
\text { A. Jaeger }\end{array}$ & & & & + & Neotropical & BA. \\
\hline $\begin{array}{l}\text { Trichosteleum papillosum (Hornsch.) A. } \\
\text { Jaeger }\end{array}$ & & + & & + & Neotropical & $\begin{array}{l}\text { AC, AM, BA, ES, MT, } \\
\text { PA, PE, RJ, RO, RR, SE, } \\
\text { SP. }\end{array}$ \\
\hline Trichosteleum sentosum (Sull.) A. Jaeger & + & + & + & + & Neotropical & BA, PA, PE, RJ. \\
\hline Trichosteleum vincentinum (Mitt.) A. Jaeger & & + & & + & Neotropical & AM, BA. \\
\hline \multicolumn{7}{|l|}{ STEREOPHYLLACEAE } \\
\hline $\begin{array}{l}\text { Pilosium chlorophyllum (Hornsch.) Mull. } \\
\text { Hal. ex Broth. }\end{array}$ & + & & + & & Neotropical & $\begin{array}{c}\text { AC, AL, AM, AP, BA, ES, } \\
\text { GO, MG, MS, MT, PA, } \\
\text { PE, RJ, RR, RO, SP. }\end{array}$ \\
\hline \multicolumn{7}{|l|}{ THUIDIACEAE } \\
\hline Pelekium scabrosulum (Mitt.) Touw & + & & & + & Neotropical & $\begin{array}{l}\text { AC, AM, BA, MT, PA, } \\
\text { PE, RO, RR. }\end{array}$ \\
\hline
\end{tabular}

\footnotetext{
* Nova ocorrência.
} 
(UFBA) foram consultadas para a complementação da listagem florística. O sistema de classificação utilizado foi o de Renzaglia et al. (2009) para as hepáticas e Goffinet et al. (2009) para os musgos. A distribuição geográfica das espécies no Brasil foi obtida a partir dos trabalhos de Yano (1981, 1989, 1995, 2006, 2008, 2011) e Costa et al. (2011).

Os espécimes identificados foram acondicionados em envelopes de papel padronizados com dimensões de $12,8 \times 9,5 \mathrm{~cm}$, seguindo a metodologia usual proposta por Yano (1984), e depositados no Herbário da Universidade Estadual de Feira de Santana (HUEFS), com duplicatas enviadas ao Herbário Alexandre Leal Costa (ALCB) da Universidade Federal da Bahia.

\section{Resultados e Discussão}

Foi registrado um total de 206 táxons infragenéricos (195 espécies e 11 variedades), 73 gêneros e 25 famílias de briófitas epífitas nos fragmentos de Floresta Atlântica da REM (tabela 1). Do total, 123 são hepáticas distribuídas em 33 gêneros e oito famílias (figura 2); e 83 musgos pertencentes a 40 gêneros e 17 famílias (figura 3). O fragmento da Mata da Vila 5 (MV) apresentou a maior riqueza específica com 117 espécies, por outro lado, o fragmento Luís Inácio (LI) foi o menos rico, com 98 espécies (figura 4). O inventário florístico possibilitou a ampliação da distribuição geográfica de seis espécies, sendo Chiloscyphus proteus (Herzog) J.J. Engel \& R.M. Schust., Calymperes levyanum Besch., C. tenerum Mull. Hal. e Groutiella obtusa (Mitt.) Florsch. novas citações para a região Nordeste; e Chiloscyphus serratus (Mitt.) J.J. Engel \& R.M. Schust. e Callicostella merkelii (Hornsch.) A. Jaeger, primeiros registros para o Estado da Bahia. As famílias mais representativas foram Lejeuneaceae (84 spp.), Calymperaceae (20 spp.), Plagiochilaceae (14 spp.), Sematophyllaceae (12 spp.) e Radulaceae (7 spp.). As famílias com menor riqueza específica foram Aneuraceae, Bryaceae, Setereophyllaceae e Thuidiaceae, com uma espécie cada. A maior representatividade das famílias Lejeuneaceae e Calymperaceae está de acordo com o que, usualmente, é observado na literatura, já que estes táxons estão entre as quatro famílias mais representativas em florestas úmidas de terras baixas (Gradstein et al. 2001).

As hepáticas apresentaram uma notável maior riqueza de espécies em relação aos musgos. A família
Lejeuneaceae contribuiu expressivamente para este resultado, contendo $40 \%$ do total de espécies encontradas na REM. No entanto, sabe-se que em florestas tropicais úmidas, este percentual pode chegar a 70\% do total de espécies (Cornelissen \& Gradstein 1990, Gradstein et al. 1990, Pôrto 1990). As hepáticas apresentam-se como uma importante influência para a riqueza de briófitas em florestas tropicais (Wolf 1993); e diversos trabalhos mencionam sua dominância nesses ecossistemas (Richards 1954, Cornelissen \& Gradstein 1990, Gradstein et al. 1990, Pôrto 1992), sobretudo quando se trata de briófitas epífitas (Campelo \& Pôrto 2007).

Os resultados obtidos atestam a significativa riqueza e diversidade de briófitas dos fragmentos de Floresta Atlântica da REM, demonstrando ainda que

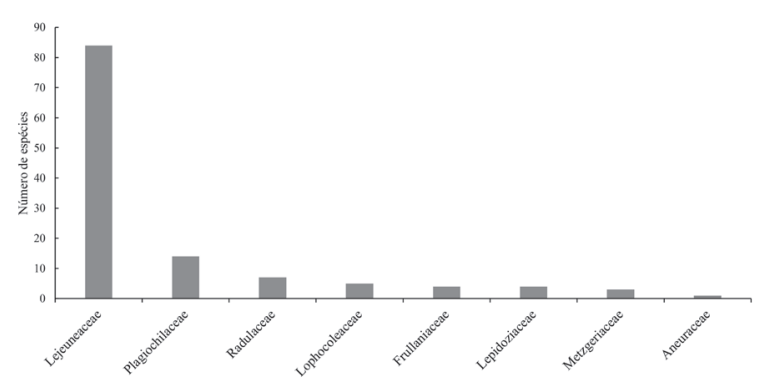

Figura 2. Riqueza específica das famílias de hepáticas epífitas nos fragmentos de Floresta Atlântica da Reserva Ecológica Michelin no Estado da Bahia, Brasil.

Figure 2. Species richness of epiphytic liverwort families in forest fragments of the Reserva Ecológica Michelin in Bahia State, Brazil.

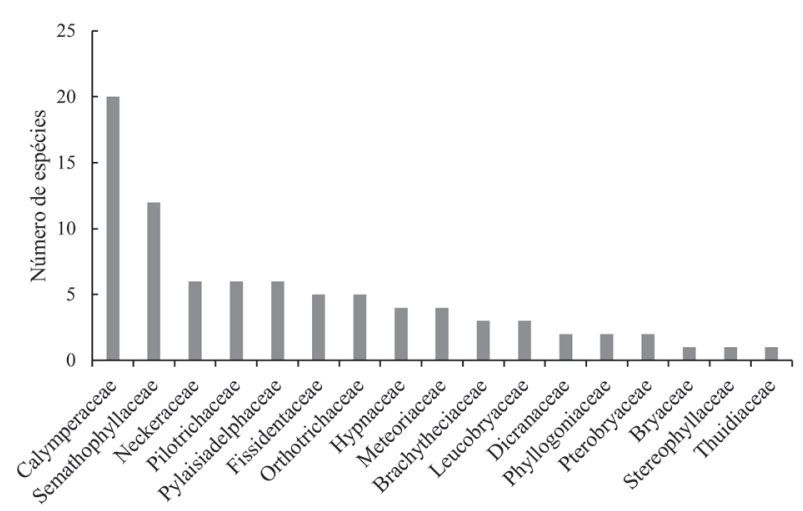

Figura 3. Riqueza específica das famílias de musgos epífitos nos fragmentos de Floresta Atlântica da Reserva Ecológica Michelin, Estado da Bahia, Brasil.

Figure 3. Species richness of epiphytic moss families in forest fragments of Reserva Ecológica Michelin in Bahia State, Brazil. 


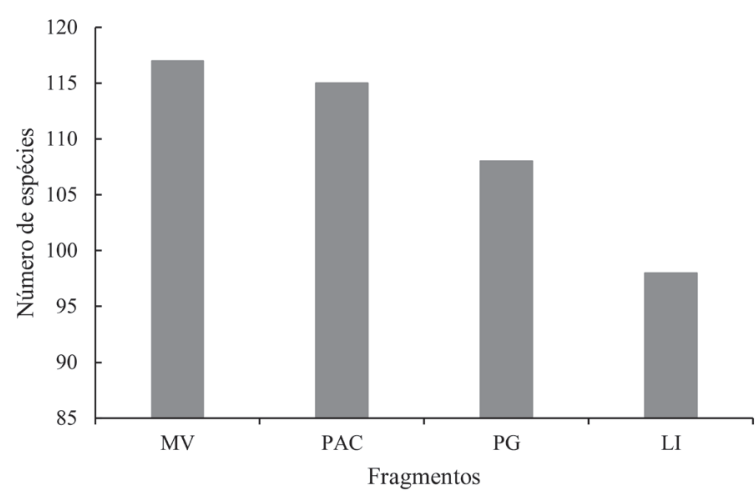

Figura 4. Riqueza específica de briófitas epífitas nos fragmentos de Floresta Atlântica da reserva ecológica Michelin, Estado da Bahia, Brasil.

Figure 4. Species richness of epiphytic bryophytes in forest fragments of Reserva Ecológica Michelin in Bahia State, Brazil.

os mesmos estão dentro dos padrões mencionados para as florestas tropicais úmidas, em termos de composição florística, tendo em vista que já entre as famílias encontradas estão aquelas que representam cerca de $90 \%$ das briófitas encontradas neste tipo de ecossistema: Pilotrichaceae, Calymperaceae, Dicranaceae, Fissidentaceae, Frullaniaceae, Hypnaceae, Lejeuneaceae, Lepidoziaceae, Meteoriaceae, Neckeraceae, Orthotrichaceae, Plagiochilaceae, Pterobryaceae, Radulaceae e Sematophyllaceae (Gradstein \& Pócs 1989).

\section{Agradecimentos}

Os autores agradecem à Coordenação de Aperfeiçoamento de Pessoal de Nível Superior CAPES, pela bolsa de doutorado concedida ao primeiro autor; à direção da REM, pela disponibilização de infra-estrutura e logística para a realização dos trabalhos de campo; ao Dr. Paulo Eduardo Aguiar Saraiva Câmara, da Universidade de Brasília (UnB), pela confirmação de espécies de Taxithelium.

\section{Literatura citada}

Angely, J. 1961. Musgos Paranaenses: contribuição para o estudo e conhecimento da flora briológica do Paraná. Revista do Instituto Paranaense de Botânica 20: 1-7.

Angely, J. 1968. Bryophytos Paranaenses: In: Flora Analítica do Paraná, Curitiba. Phyton 7: 55-91.

Bastos, C.J.P. 2011. Cheilolejeunea ornata (Lejeuneaceae), a new species from Brazilian Atlantic Forest. Journal of Bryology 33: 86-88.
Bastos, C.J.P. \& Gradstein, S.R. 2006. Two new species of Cheilolejeunea (Spruce) Schiffn. (Lejeuneaceae) from Brazil: C. lacerata sp. nov. and C. rupestris sp. nov. Journal of Bryology 28: 133-138.

Bastos C.J.P. \& Yano, O. 2002. Pycnolejeunea porrectilobula (Lejeuneaceae), a new species from Brazil. Nova Hedwigia 74: 439-443.

Bastos, C.J.P. \& Yano, O. 2004. New records of Lejeuneaceae (Marchantiophyta) for the Brazil. Acta Botanica Malacitana 29: 13-21.

Bastos, C.J.P. \& Yano, O. 2005. Notes on the occurrence of Cheilolejeunea paroica Mizut. (Lejeuneaceae: Marchantiophyta) in Neotropics. Acta Botanica Malacitana 30: 7-10.

Bastos, C.J.P. \& Yano, O. 2009. O gênero Lejeunea Libert (Lejeuneaceae) no Estado da Bahia, Brasil. Hoehnea 36: 303-320.

Bastos, C.J.P., Cerqueira, A.S. \& Yano, O. 2003. Musgos de floresta higrófila e savana gramíneo-lenhosa do Recôncavo, Bahia, Brasil. Revista Brasileira de Biociências 8: 35-48.

Buck, W.R. 1998. Pleurocarpous Mosses of the West Indies. Memoirs of the New York Botanical Garden 82: 1-400.

CEI/CONDER. 1993. Informações básicas dos municípios baianos: Região Litoral Sul. Centro de Estatística e Informação/Companhia do Desenvolvimento da Região Metropolitana de Salvador. Secretaria do Planejamento, Ciência e Tecnologia, Salvador, Bahia.

Campelo, M.J.A. \& Pôrto, K.C. 2007. Brioflora epífita e epífila da RPPN Frei Caneca, Jaqueira, PE, Brasil. Acta Botanica Brasilica 21: 185-192.

Cornelissen, J.H.C. \& Gradstein, S.R. 1990. On the occurrence of Bryophytes and macrolichens in different lowland rain forest types at Mabura Hill, Guyana. Tropical Bryology 3: 29-35.

Costa, D.P., Pôrto, K.C., Luizi-Ponzo, A.P., Ilkiu-Borges, A.L., Bastos, C.J.P., Câmara, P.E.A.S., Peralta, D.F., Vilas Bôas-Bastos, S.B.V., Imbassahy, C.A.A., Henriques, D.K., Gomes, H.C.S., Rocha, L.M., Santos, N.D., Siviero, T.S., Vaz-Imbassahy, T.F. \& Churchill, S.P. 2011. Synopsis of the Brazilian moss flora: checklist, distribution and conservation. Nova Hedwigia 93: 277-334.

Costa, D.P. \& Silva, A.G. 2003. Briófitas da Reserva Natural da Vale do Rio Doce, Linhares, Espírito Santo, Brasil. Boletim do Museu de Biologia Mello Leitão 16: 21-38.

Costa, D.P. \& Yano, O. 1995. Musgos do município de Nova Friburgo, Rio de Janeiro, Brasil. Arquivos do Jardim Botânico do Rio de Janeiro 33: 99-118.

Costa, D.P., Imbassahy, C.A.A. \& Silva, V.P.A.V. 2005. Checklist and distribution of mosses, liverworts and hornworts of Rio de Janeiro State, Brazil. The Journal of the Hattori Botanical Laboratory 98: 259-298. 
Dauphin, G. 2003. Ceratolejeunea. Flora Neotropica Monograph 90: 1-86.

Florschütz, P.A. 1964. The mosses of Suriname. E.J. Brill, Leiden.

Forzza, R.C. Leitman, P.M., Costa, A.F., Carvalho Jr., A.A., Peixoto, A.L., Walter, B.M.T., Bicudo, C., Zappi, D., Costa, D.P., Lleras, E., Martinelli, G., Lima, H.C., Prado, J., Stehmann, J.R., Baumgratz, J.F.A., Pirani, J.R., Sylvestre, L., Maia, L.C., Lohmann, L.G., Queiroz, L.P., Silveira, M., Coelho, M.N., Mamede, M.C., Bastos, M.N.C., Morim, M.P., Barbosa, M.R., Menezes, M., Hopkins, M., Secco, R., Cavalcanti, T.B. \& Souza, V.C. 2010 Introdução. In: Lista de Espécies da Flora do Brasil. Jardim Botânico do Rio de Janeiro. Disponível em: http://floradobrasil. jbrj.gov.br (acesso em: IV-2013).

Fulford, M.H. 1963. Manual of the leafy Hepaticae of Latin América I. Memoirs of the New York Botanical Garden 11: 1-172.

Fulford, M.H. 1966. Manual of the leafy Hepaticae of Latin América II. Memoirs of the New York Botanical Garden 11: 173-276.

Fulford, M.H. 1968. Manual of the leafy Hepaticae of Latin América III. Memoirs of the New York Botanical Garden 11: 277-392.

Fulford, M.H. 1976. Manual of the leafy Hepaticae of Latin América I. Memoirs of the New York Botanical Garden 11: 393-535.

Germano, S.R. \& Pôrto, K.C. 1996. Floristic survey of epixilic bryophytes of an area remmant of the Atlantic Forest (Timbaúba-PE, Brazil). 1. Hepaticopsida (Except Lejeuneaceae) and Bryopsida. Tropical Bryology 12: $21-28$.

Giancotti, C. \& Vital, D.M. 1989. Flora briofítica da Reserva do Alto da Serra de Paranapiacaba, São Paulo: 1 - Lejeuneaceae (Hepaticopsida). Acta Botanica Brasilica 3(supl.): 169-177.

Goffinet, B., Buck, W.R. \& Shaw, A.J. 2009. Morphology and classification of the Bryophyta. In: B. Goffinet \& A.J. Shaw (eds.). Bryophyte Biology; Cambridge University Press, v. 2, pp. 55-138.

Gradstein, S.R. \& Costa, D.P. 2003. The Hepaticae and Anthocerotae of Brazil. Memoirs of the New York Botanical Garden 87: 1-318.

Gradstein, S.R. \& Pócs, T. 1989. Bryophytes. In: H. Leith \& M.J.A. Werger (eds.). Tropical rain forest ecosystems. Elsevier Science, Amsterdam, pp. 311-325.

Gradstein, S.R., Churchill, S.P. \& Salazar Allen, N. 2001. Guide to the bryophytes of tropical America. New York Botanical Garden Press, New York.

Gradstein, S.R., Montfoort, D. \& Cornelissen, J.H.C. 1990. Species richness and phytogeography of the bryophyte flora of the Guianas, with special reference to the lowland forest. Tropical Bryology 2: 117-126.
Hirai, R.Y., Yano, O. \& Ribas, M.E.G. 1998. Musgos da mata residual do Centro Politécnico (Capão da Educação Física), Curitiba, Paraná, Brasil. Boletim do Instituto de Botânica 11: 81-118.

Kummrow, R. \& Prevedello, S.M. 1982. Lista de musgos paranaenses do Museu Botânico municipal. Boletim do Museu Botânico Municipal Curitiba 54: 1-36.

Marinho, M.G.V. \& Mariz, G. 1992. Addition to the moss flora of remaining area of the Atlantic Forest (Mata de Dois Irmãos), Recife-PE, Brasil. Biologica Brasilica 4: 9-22.

Oliveira-e-Silva, M.I.M.N. \& Yano, O. 2000a. Anthocerotophyta e Hepatophyta de Mangaratiba e Angra dos Reis, Rio de Janeiro, Brasil. Boletim do Instituto de Botânica 13: 1-102.

Oliveira-e-Silva, M.I.M.N. \& Yano, O. 2000b. Musgos de Mangaratiba e Angra dos Reis, Rio de Janeiro, Brasil. Boletim do Instituto de Botânica 14: 1-137.

Oliveira-e-Silva, M.I.M.N., Milanez, A.I. \& Yano, O. 2002. Aspectos ecológicos de briófitas em áreas preservadas de Mata Atlântica, Rio de Janeiro, Brasil. Tropical Bryology 22: 77-102.

Pôrto, K.C. 1990. Bryoflores d'une forêt d'altitud moyenne dans l'État de Pernambuco (Brésil). 1. Analyse floristique. Cryptogamie, Bryologie et Lichénologie 11: 109-161.

Pôrto, K.C. 1992. Bryoflores d'une forêt de plaine et d'une forêt d'altitude moyenne dans l'État de Pernambuco (Brésil); Analyse écologique comparative des forêts. Cryptogamie, Bryologie et Lichénologie 13: 187-219.

Renzaglia, K.S., Villareal, J.C. \& Duff, R.J. 2009. New Insights into Morphology, Anatomy, and Systematic of Hornworts. In: B. Goffinet \& A.J. Shaw (eds.). Bryophyte Biology. Cambridge University Press, New York. pp. 138-171.

Richards, P.W. 1954. Notes on the bryophyte communities of Lowland Tropical Rain Forest, with special reference to Moraballi Creek, British Guyana. Vegetatio 5-6: 319-328.

Sehnem, A. 1969. Musgos Sul-brasileiros 1. Pesquisas, Série Botânica 27: 1-41.

Sehnem, A. 1970. Musgos Sul-brasileiros 2. Pesquisas, Série Botânica 28: 1-117.

Sehnem, A. 1972. Musgos Sul-brasileiros 3. Pesquisas, Série Botânica 29: 1-70.

Sehnem, A. 1976. Musgos Sul-brasileiros 4. Pesquisas, Série Botânica 30: 1-79.

Sehnem, A. 1978. Musgos Sul-brasileiros 5. Pesquisas, Série Botânica 32: 1-170.

Sehnem, A. 1979. Musgos Sul-brasileiros 6. Pesquisas, Série Botânica 33: 1-149.

Sehnem, A. 1980. Musgos Sul-brasileiros 7. Pesquisas, Série Botânica 34: 1-121. 
Sharp, A.J., Crum, H. \& Eckel, P.M. (eds). 1994. The moss flora of Mexico. Memoirs of the New York Botanical Garden 69: 1-1113.

Schäfer-Verwimp, A. 1989. New or interesting records of Brazilian Bryophytes. The Journal of the Hattori Botanical Laboratory 67: 313-321.

Schäfer-Verwimp, A. 1991. Contribution to the knowledge of the bryophyte flora of Espírito Santo, Brazil. The Journal of the Hattori Botanical Laboratory 69: 147-170.

Schäfer-Verwimp, A. 1992. New or interesting records of Brazilian bryophytes, III. The Journal of the Hattori Botanical Laboratory 71: 55-68.

Valente, E.B. \& Pôrto, K.C. 2006a. Novas ocorrências de hepáticas (Marchantiophyta) para o estado da Bahia, Brasil. Acta Botanica Brasilica 20: 1-7.

Valente, E.B. \& Pôrto, K.C. 2006b. Hepáticas (Marchantiophyta) de um fragmento de Mata Atlântica na Serra da Jibóia, município e Santa Teresinha, Bahia, Brasil. Acta Botanica Brasilica 20: 433-441.

Veloso, H.P., Rangel Filho, A.L.R. \& Lima, J.C. 1991. Classificação da vegetação brasileira, adaptada a um sistema universal. IBGE. Rio de Janeiro.

Vilas Bôas-Bastos, S.B. 2009. Hypnella symphyodontoides (Bryophyta: Pilotrichaceae), a new species from Brazil. Journal of bryology 31: 20-23.

Vilas Bôas-Bastos, S.B. \& Bastos, C.J.P. 2000. New occurrence of pleurocarpous mosses for the state of Bahia, Brasil. Tropical Bryology 18: 65-73.

Vilas Bôas-Bastos, S.B. \& Bastos, C.J.P. 2002. Occurrence of the genus Pilotrichum P. Beauv. (Pilotrichaceae, Bryopsida) in the state of Bahia, Brazil. Nova Hedwigia 75: 217-225

Visnadi, S.R. 2002. Meteoriaceae (Bryophyta) da Mata Atlântica do estado de São Paulo. Hoehnea 29: 159-187.

Visnadi, S.R. 2005. Brioflora de Mata Atlântica do Estado de São Paulo: região norte. Hoehnea 32: 215-231.
Visnadi, S.R. 2006. Sematophyllaceae da Mata Atlântica do Nordeste do estado de São Paulo. Hoehnea 33: 455-484.

Visnadi, S.R. \& Vital, D.M. 2000. Lista das briófitas ocorrentes no Parque Estadual das Fontes do Ipiranga PEFI. Hoehnea 27: 279-294.

Wolf, J.H.D. 1993. Epiphyte communities of tropical montane rain forest in the northern Andes. II. Upper montane communities. Phytocoenologica 22: 53-103.

Yano, O. 1981. A Checklist of Brazilian mosses. The Journal of the Hattori Botanical Laboratory 50: 279-456.

Yano, O. 1984. Briófitas. In: Técnicas de coleta, preservação e herborização de material botânico (O. Fidalgo \& V.L.R. Bononi, coords). Instituto de Botânica, São Paulo, Manual 4: 27-30.

Yano, O. 1989. An additional checklist of Brazilian bryophytes. The Journal of the Hattori Botanical Laboratory 66: 371-434.

Yano, O. 1995. A new additional annotated cheklist of Brazilian bryophytes. The Journal of the Hattori Botanic Laboratory 78: 137-182.

Yano, O. 2005. Novas ocorrências de Bryophyta para vários estados do Brasil. Acta Amazonica 34: 559-576.

Yano, O. 2006. Novas adições ao catálogo de Briófitas Brasileiras. Boletim do Instituto de Botânica 17: 1-142.

Yano, O. 2008. Catálogo de Antóceros e Hepáticas Brasileiros: literatura original, basiônimo, localidadetipo e distribuição geográfica. Boletim do Instituto de Botânica 19: 1-110.

Yano, O. 2011. Catálogo de musgos brasileiros: literatura original, basiônimo, localidade-tipo e distribuição geográfica. São Paulo: Instituto de Botânica. Disponível em: http://www.ibot.sp.gov.br/publicacoes/virtuais/ musgos $\% 20$ brasileros\%20completo\%2023-05.pdf. (acesso em I-2014).

Yano, O. \& Colletes, A.G. 2000. Briófitas do Parque Nacional de Sete Quedas, Guaíra, PR, Brasil. Acta Botanica Brasilica 14: 215-242. 